\title{
Trends in Gastrointestinal Diseases in Japan
}

The 11th Annual Meeting of the Japanese Gastroenterological Association (JGA) was organized by Prof. Hisao Tajiri and held in Tokyo on February 13 and 14, 2015. During this conference, 4 core symposia, 1 international symposium, 7 educational lectures, 12 workshops, and 1 forum were held among other events. The 4 themes of the core symposia were as follows: (1) therapeutic strategies in gastrointestinal cancers, (2) post-surgical issues in inflammatory bowel disease (IBD), (3) new horizons in functional dyspepsia, and (4) endoscopic diagnosis and treatment of small intestinal lesions.

The articles published in the present Digestion JGA Special Issue 2016 represent the best presentations from the 11th Annual Meeting. The session chairpersons and editorial board members recommended numerous papers from their sections; subsequently, the editorial committee of the JGA selected distinguished presentations from these recommendations and invited the authors to submit their papers for this special issue. Six reviews and four original articles were accepted for the final publication. All manuscripts were independently reviewed by two members of the JGA editorial committee.

Gastric cancer is a major cause of cancer deaths worldwide. The incidence rate of gastric cancer is especially high in the East Asian countries, including Japan. Infection caused by Helicobacter pylori (H. pylori) plays an important role in gastric carcinogenesis. For the early detection of gastric cancer, screening programs have been implemented in Japan and other high-risk areas. One review in this special issue focuses on gastric cancer screening by a combined assay for serum anti- $H$. pylori IgG antibodies and serum pepsinogen levels. In Japan, endoscopic submucosal dissection (ESD) has been widely performed for the treatment of gastric mucosal cancer. An original article describes the clinicopathological outcome of patients with early gastric cancer after non-curative ESD. Pangastritis and corpus-predominant gastritis caused by H. pylori infection are highly prevalent in Japan. In patients with these types of gastritis, eradication of $H$. $p y$ lori causes increased acid secretion, which may influence the incidence of acid-related diseases such as gastroesophageal reflux disease (GERD). Two reviews focus on the incidence of such diseases after the eradication of $H$. pylori in the Japanese population.

The incidences of GERD, Barrett's esophagus, and Barrett's esophageal adenocarcinoma are lower in Japan and other Asian countries than those in the Western countries. GERD has been recently increasing in Japan, which may increase the future incidence of Barrett's esophageal adenocarcinoma. Currently, in Japan, Barrett's esophageal adenocarcinoma arising from short-segment Barrett's esophagus is more prevalent than that arising from long-segment Barrett's esophagus. Another article in this issue describes the conventional white light endoscopic features of small superficial Barrett's esophageal adenocarcinoma.

Eosinophilic esophagitis is a clinicopathological disease characterized by symptoms related to esophageal dysfunction and eosinophil-rich infiltrate in the esophageal epithelium. Incidences of eosinophilic esophagitis

\section{KARGER}

E-Mail karger@karger.com

www.karger.com/dig
(C) 2016 S. Karger AG, Basel

0012-2823/16/0931-0005\$39.50/0 
are gradually increasing in Japan. In this issue, a review describes the recent progress in research on eosinophilic esophagitis and gastroenteritis.

IBD, which includes ulcerative colitis and Crohn's disease, has been increasing continuously over the past several decades in Japan, although the prevalence of IBD is still lower in Japan than that in the Western countries. Aberrant immune responses to gut microbiota in genetically susceptible hosts are considered to be major contributors in IBD pathogenesis. Life style changes, such as change in diet habit, and subsequent alteration in the gut microbiota, may result in the increasing prevalence of IBD in Japan. In another article of this issue, the reduced abundance of butylate-producing bacteria species is observed in the fecal microbiota in Crohn's disease. Activation of NF- $\kappa \mathrm{B}$ induces the transcriptional activation of a variety of genes that mediate immune and inflammatory responses. One review of this issue focuses on the activation of NF- $\kappa \mathrm{B}$ in IBD and colitis-associated carcinogenesis. Another review focuses on prostaglandin urinary metabolites as biomarkers for inflammation in ulcerative colitis. $\mathrm{Mu}$ cosal healing or endoscopic remission has emerged as a desirable treatment goal in Crohn's disease. Endoscopic severity predicting the long-term prognosis of Crohn's disease patients with clinical remission is described in another article.

We believe this issue will provide useful information for clinicians and investigators. We wish to express our sincere gratitude to all the authors, chairpersons, and editorial board members for their contributions to this special issue.

\section{Disclosure Statement}

Y. Shinomura is a co-editor-in-chief of Digestion and the acting editor for the publication of this special issue sponsored by JGA. $\mathrm{He}$ is a member of the Board of Directors of JGA and serves as the JGA Editorial Representative for Digestion, the official journal of JGA.

Yasuhisa Shinomura, MD, $\mathrm{PhD}$ Chairperson of the JGA Editorial Committee 